\title{
Organization of Computations in Conditions of Limited Amount of Content Addressable Memory in the Parallel Dataflow Computing System "Buran"
}

\author{
N.N. Levchenko, A.S. Okunev, D.N. Zmejev, A.V. Klimov, A.L. Stempkovsky \\ Institute for Design Problems in Microelectronics of RAS, Moscow nick@ippm.ru, oku@ippm.ru, \\ zmejevdn@ippm.ru, aklimov@ippm.ru
}

\begin{abstract}
The parallel dataflow computing system "Buran" implements the dataflow computing model with a dynamically formed context. To ensure effective parallelization of tasks, it is necessary to organize the operation of the system in conditions of a limited amount of content addressable memory of keys, which is part of the computational core. The article describes the composition and operation of the parallel dataflow computing system, problems associated with the specifics of the computation organization in this system, as well as methods of computation organization to eliminate the possibility of overflow of the content addressable memory of keys the computational core of the system. Such methods are: the computation organization method using a data input block; the method using token-"lock"; the method using division of tokens into stages and swapping out "passive" stages into external storage; the method using "postponed" stages only. These methods allow the solution of tasks with a large amount of data, by having a limited amount of the content addressable memory in the parallel dataflow computing system.
\end{abstract}

Keywords - organization of computations, parallel dataflow computing system, division into stages, data input block, token swapping.

\section{INTRODUCTION}

Recently, researchers are increasingly beginning to show interest in dataflow computing. Modern projects whose computing model uses the dataflow principle (mainly "static dataflow architectures") are WaveScalar, TRIPS, Merrimac, and a number of others. Also the Maxeler computing system is being developed, in which the accelerator is built on the principles of static dataflow [1]. The use of a dataflow computing model can be relevant in the development of supercomputers [2].

Earlier (three decades ago) work was carried out on the implementation of dataflow computing systems [3-4]. However, due to unresolved significant problems, including those associated with the implementation of content addressable memory, they were discontinued. Now the application of the dataflow computing model is especially important in the development of modern supercomputers.

IPPM RAS is working on the development of the dataflow computing model with a dynamically formed context and developing the architecture of the parallel dataflow computing system (PDCS), which implements this computing model [5].

The limited amount of content addressable memory of keys of the matching processor is one of the most serious problems in creating high-performance dataflow computing systems. This problem is connected with the peculiarities of the computing model, the need for a quick comparison of data when initiating computational operations, and also with technological limitations when using content addressable memory. To solve this and other problems, methods of organizing computations with a limited amount of content addressable memory were developed, which allow not only to control the behavior of computational processes in the system and distribution to the computational cores of the system, but also to prevent overflow of content addressable memory of keys.

\section{PARALLEL DATAFLOW COMPUTING SYSTEM}

The PDCS "Buran" [6]-[8] is a multi-core scalable computing system. The computational core includes an execution unit, a matching processor, a token commutator, and a hashing unit. Units of information are transmitted in the form of tokens between the cores in the system. The token is a message containing, in addition to the operand, a set of service fields and a tag - key. Switching between the cores is carried out according to the index of the computational core, which is generated in the hashing unit by the programmer's distribution function based on token fields. The data input block (DIB) is responsible for data entry into the computing system [9]. The matching processor [10]-[11] in the PDCS provides interaction of tokens of various types by comparing the token key fields taking into account the multiplicity and evaluating the state of other token fields.

The token, as an information structural unit, contains: data (operand), key, key mask, multiplicity and set of service fields. Both between the cores in the group, and between groups of cores, the same interaction protocol (that delivers tokens) operates. Due to this, the system can be unlimitedly scaled.

\section{Each core of a computing system consists of:}

- the matching processor (MP), in which there is a matching of tokens according to certain rules; 
- the execution unit (EU), in which the program of node is executed;

- the hashing unit, in which the distribution function is calculated at the hardware level, which determines the number of the target core for the token.

Computational cores, in turn, are combined into a computational module, which can be implemented as a crystal. Computational modules are interconnected by a switching network.

In general, a computing system that implements a dataflow computing model has the following features that support high-performance computing:

- there is a content addressable memory (CAM) with an advanced command system, on the basis of which the global virtual address space (keys of tokens, or virtual nodes) is implemented;

- the system is well scaled, allowing the possibility of creating a multi-core crystal, as well as highperformance systems based on these crystals;

- implemented hardware detection of implicit parallelism of the task during its solution;

- there are hardware and software tools for controlling task parallelism;

- there is asynchronous operation of individual units of the system;

- dataflow computing process organization allows to level the delays in the communication network.

When creating the PDCS, the complex of hardwaresoftware methods and tools for efficient organization of computations has been developed. Software tools include the use of hash functions and the "distribution" paradigm [7]; the matching processor refers to hardware.

The matching processor functions as a computation control device [12]. The MP, in particular, compares the keys of the task tokens to each other and solves problems associated with overflow or underutilization of hardware resources in the process of computation.

The main functions of the MP are as follows:

- activation of calculations by data readiness;

- synchronization of computational processes by data;

- organizing a group sample of operands by mask;

- computing resources management using special operations;

- monitoring the state of hardware resources and the intensity of computing processes.

The MP includes a content addressable memory of keys (CAMK) in which keys are matched, responses are fixed, cells busy statuses are set and addresses of free cells are formed.
Using the CAMK of the MP allows the extraction from the task all the parallelism that exists in its program. That allows the efficient utilization of the available hardware resources of the computing system and the achievement of a high degree of task scaling.

The amount of the CAMK of each computational core is limited, due to the MP technology and other architectural features of the system. In view of this, there is a possibility of the CAMK overflow during the computing system operation.

The CAMK overflow is associated with the situation when after processing of each received token more than one token is generated in the EU. Since the selection of tokens for processing is a random process, the average residence time of the token key in the CAMK increases. If no action is taken, then the average time to waiting the "pair" for the token will increase. The possibility of overflowing the CAMK is affected by:

- the multiplicity of tokens;

- insufficient number of EUs per MP or processing of a large program node by EUs;

- inefficient distribution function.

At the very beginning of the project, the global CAM of the system was a separate unit with a single structure of associative cells. In the course of the work, the shortcomings of such an organization of the memory system were revealed, and at the next stages of the work, the CAMK was studied with various options for implementing such a memory.

\section{PROBLEMS RELATED TO THE ORGANIZATION OF COMPUTATIONS IN THE PDCS}

When creating the parallel dataflow computing system "Buran", considerable attention was paid to the process and means of organizing computations in the system. This is due, firstly, to the requirement to reduce the load on the switching network of the computing system; secondly, to the need to balance the utilization of computational cores of the system for efficient use of hardware; and thirdly, to the limited volume of hardware CAM located in the computational cores of the system.

Standard methods of organizing computations used in traditional computing systems are not suitable for the PDCS, since it implements a dataflow computing model with a dynamically formed context.

The CAMK overflow in the process of computation can lead to blocking of the computational process, which determines the relevance of the developed hardware and software methods for organizing computations.

The specificity of the organization of computations in the PDCS is that programming is carried out in the "distribution" paradigm, in contrast to the "gathering" paradigm used in the traditional computing model.

Particular attention in the organization of computations in such a system has to be paid to the CAM, the hardware 
implementation of which implements the dataflow principle in the most efficient way. However, due to the peculiarities of such a memory, both technological and principal (access by content, not by address), its size, as mentioned above, is limited (significantly less than that of direct addressable memory of traditional systems). At the same time, the problem of CAMK overflow by the keys of tokens that are waiting for interaction with other tokens is one of the main problems for all dataflow systems.

Equally important are the issues related to the need to manage the level of parallelism, both during its explosive growth and its sharp drop, when the available hardware resources become idle due to the lack of matching tokens ready for processing on the system execution units.

Attention should also be paid to the distribution of tokens between the CAM modules belonging to different computational cores of the system, which, in fact, form the global CAMK.

In addition, it is necessary to minimize the exchange of tokens between the computational cores and balance the load on the computational cores of the system.

To solve the above problems, several methods have been developed that are associated with both different methods of data entry into the system, and using different approaches to token swapping in the CAMK.

\section{COMPUTATION ORGANIZATION METHODS}

In this section, four methods of organizing computations in the PDCS will be described. The first method is associated with "intelligent" data input into the system. For this method, various data input algorithms have been developed, depending on the task type. The method allows the selection of one or another hash function for grouping tokens, supports the process of token swapping in the CAM and thus defuses the problem of limiting the amount of this memory in computational cores and the system as a whole.

Three other methods are related to the use of token swapping. The essence of these three computation organization methods on the one hand is similar to the standard data swapping used in traditional computing systems. However, the most important part of these methods is completely different. This important part concerns how to determine what kind of data and at what point to swap. These problems are closely related to the nature of CAM, which is accessed not by address, but by content. Since the data in such a memory is written to any free space, their packaging (block record) is difficult to implement.

\section{A. Computation organization method using DIB}

The data input block in the PDCS not only organizes the control of data input in order to increase the efficiency of computations in a system with a limited resource of CAMK, but also performs recoding of data coming from outside (from the host machine or external sensors).

Data from the host machine enters the input data buffer of the DIB. Further, in the token generator, in accordance with the specified templates, all token fields are filled.
At the next stage, the tokens arrive at the token input node, in which the user chooses a hash function from the existing ones, determines its parameters or sets its own hash function, thereby calculating the number of the subset of tokens and the index of the computational core. In the same node, an analysis is made as to whether the tokens should be delayed in the DIB or sent to the token output buffer for transferring to the token commutator of the computing system.

For DIB, data input algorithms were developed that allow not only to form tokens from the transmitted data, but also to arrange the order of receipt of tokens in the computing system in the most efficient way for each specific task.

For example, in the "input of sparse data" mode that can be shared with any other modes, tokens with only nonzero data are sent to the computing system, which allows one of the features of the parallel dataflow computing system to be implemented - no need to store "extra" data which is not processed. It is also possible to apply a mask on the "data" field. The masked bytes are considered null.

The "portion input" mode is used in conjunction with other data input modes. For example, when a task is divided into temporal stages, only a limited number of stages can be "active". In this case, it is worthwhile to send only the data that related to the "active" stages into the computing system, and the remaining stages - to delay in the DIB. This allows significantly reduce the amount of CAM required to perform the task. Also, by changing the size of the "portion" of data coming from the DIB, the parallelism of the task can be adjusted. One possible implementation is to count the number of sent and received tokens. After sending a specified number of tokens, DIB pauses input until the results of their processing are received, and then sends the next "portion" of tokens.

Some DIB nodes can be used to construct a block for data swapping, which is part of the hierarchical CAM of the computing system.

The study of the influence of various data input algorithms on the efficiency of tasks execution on the PDCS and on the required amount of the CAMK of the matching processor is also associated with the possibility of starting the computations without waiting for the load of the total program input data volume. For this, various data input algorithms were developed.

\section{B. Computation organization method using token swap- ping in the dynamic mode}

The work of the swapping method is based on a special token-"lock", which functions as follows. When entering the CAMK with infinite multiplicity, the token-"lock" swaps out (moves tokens from CAM to the external storage) all matched tokens, and it remains in the CAMK to wait for the rest of the tokens associated with it (within the same range). The newly arrived tokens will be compared with the token-"lock" and swapped out if matched. The programmer determines the ranges of swapped tokens by selecting the appropriate key and mask of the token-"lock". 
A separate statistics collection unit is introduced into the MP, which collects information on the number of tokens for each of the ranges, as well as for loading of the CAMK. Also, the programmer determines the triggering thresholds for swapping: the upper threshold - upon reaching this number of tokens in the CAMK, one of the token ranges will be swapped out (unloaded from the CAMK); and the lower threshold - upon reaching this value, the earlier swapped out token ranges will be swapped in (loaded to the CAMK). By default, a certain averaged value is set as the threshold value, determined by the results of tasks executions.

This method is not able to completely solve the problem of the CAMK overflow. In addition, when creating a large-scale CAMK when moving to the next level of the hierarchy, the possibility of associative search at all subsequent levels is lost, that is, tokens are swapped out as if into a "passive" memory by commands from the control device, from which they must be returned at some point into work for later comparison. Additional information from the programmer (compiler) is also required to generate the "locks".

\section{Computation organization method using active/passive stages}

Another method of organizing computations is distribution in time using temporal stages. A temporal stage is a group of tokens, which is used when performing a certain interval of calculations - a stage. Usually such a group of tokens is associated with a rather separate part of the program (for example, iterations in the program can be designed as temporal stages).

The programmer divides the task into stages by applying hash functions that can be configured directly for the executed task using various parameters. In the process of task execution, the hash functions assign certain numbers to the stages, with which the corresponding hardware operates later by "placing" these stages. All stages are divided into "active" (the tokens are processed in the CAMK) and "passive" (the tokens are suspended in the MP - the MP delays their processing).

There are a number of requirements for the procedure of dividing into stages and for the size of the stage:

- stages with large numbers should not generate tokens with smaller stage numbers;

- the size of the stage should not exceed $10 \%$ of the amount of the CAMK;

- 6-7 stages can be "active" at the same time; after the completion of processing one of them, the next is activated, etc.

Each token, entering the MP, goes through a hashing procedure, after which it is assigned the number of the stage at which it will be claimed. After this, a check is made to which stage type the token belongs to. If its stage is "active" - the standard work follows (the token is moved to the CAMK for processing). If its stage is "passive" - the token is postponed. If the number of occupied CAMK cells exceeds the swapping threshold, then one of the active stages (with the highest number) is deactivated and its tokens are swapped out. When space in the CAMK is released to perform the postponed stage, its activation is performed.

The method can function without setting the swapping thresholds. In this case, the swapping of the stages occurs automatically when the CAMK is filled up to the level, for example, $85-90 \%$ of the total amount of the CAMK in accordance with the existing priorities of the tasks or numbers of the stages.

At the hardware level, to perform this method a block for dividing into stages is added - a hashing unit, a table of stages, a memory of postponed tokens, and a memory of swapped out tokens.

The transfer of stages from the "active" to the "passive" state and vice versa is carried out by the matching processor.

\section{Computation organization method using postponed stages}

The fourth method of organizing computations is in some way an extension of the previous one, in which, despite the fact that the required size of one stage was specified, the hardware could level out some error by swapping out an additional stage. In the method using only "postponed" stages, it was decided to abandon the "swapped out" stages, and in general - from the swapping procedure itself. When using this method, the stages will not be swapped out if there is a threat of the CAMK overflow, since this threat is minimized. Thus, only tokens of "active" stages and tokens of "postponed" stages will be involved in the computations

The table of stages is replaced with the table of "active" stages. The table of "active" stages operates according to an associative principle. It contains only the numbers of "active" stages.

A token arriving at the input of the MP goes through the procedure of obtaining the number of the stage to which it belongs. After this, the tokens of the stages that are not "active" (their stage numbers are absent in the table of "active" stages) remain at the CAMK input in the memory of the "postponed" stages. The memory of the "postponed" stages has a hierarchical structure.

The requirements for the size of the stage remain the same - about $10 \%$ of the amount of the CAMK. The user can himself limit the number of simultaneously working "active" stages, or provide this choice to the hardware. As soon as one of the stages is completed, the status of next (by the number) "postponed" stage is changed to the "active" and its tokens are sent to the CAMK input.

In this method, only the threshold for "swapping in" or activation threshold is set. In practice, the activation threshold may be the number of simultaneously running "active" stages. The threshold for "swapping in" can also be used as an additional mechanism, which, analyzing the load on the CAMK, may decide to increase the number of 
"active" stages. "Postponed" stages can go into the category of "active" by control signals from the control unit of the matching processor.

\section{E. Comparison of the proposed methods}

The method of organizing data input shows a high degree of variability and low overhead costs for its implementation, since there is no need to duplicate hardware units into each of the computational cores of the system. At the same time, its use allows the significant (up to one order of magnitude) reduction of the data amount circulated simultaneously in the computing system. It also agrees well with other computation organization methods.

The advantages of the second method are as follows: simple hardware support for this method (the introduction of the token-"lock" is sufficient to ensure the operation of this method); no suspension of the computational process during swapping; reducing the required amount of the CAMK for the tasks execution. The disadvantages of the method are: the difficulty of determining the tokens range that is not multiple of 2 , since it is determined by the bitwise key with the mask; difficulty in assigning thresholds in the CAMK for tokens swapping, which in some situations can lead to "trashing".

The third method can be considered the most universal method of computation organization in time, since it combines work with "postponed" and "swapped out" stages. However, this method requires hardware complication, in particular, the introduction of a block for hashing keys of tokens.

The fourth method has a simpler algorithm of operation and at the same time the continuity of the computational process with minimal losses in performance will be ensured. The disadvantages include the need to hash keys of tokens, as well as the fact that the programmer must ensure that the size of all "active" stages does not exceed the volume of the CAMK.

\section{CONCLUSION}

The article presents the main computation organization methods for the PDCS "Buran", associated both with data input into the system and with the use of data swapping technology in the matching processor. The implementation of these methods in the composition of software-hardware tools of the PDCS allows the solution of problems of a wide range of applications in conditions of a limited amount of CAM.

In fact, these methods eliminate the blocking of the computational process during the tasks execution.

The basic algorithms of the functioning of the hardware solutions for these methods were tested on the PDCS program model. It can be concluded that the main approach for organizing computations in the PDCS is to localize these computations in time (by using user-defined hash functions). As a result, groups of tokens (stages) are formed, which are used in the process of data input and data swapping in the CAMK.
The first method is associated with "intelligent" data input into the system. For this method, various data input algorithms have been developed, depending on the task type.

The computation organization method using token swapping in the dynamic mode has its advantages over others, but it is not able to completely solve the problem of the CAMK overflow.

Another method of computation organization is distribution in time using temporal stages.

The fourth method of organizing computations is in some way an extension of the previous one. It has a simple algorithm of operation and ensures the continuity of the computational process with minimal losses in performance.

When creating a basic set of nodes and blocks of the matching processor, it is necessary to provide hardware support for these computation organization methods in order to implement the possibility of optimizing such a set of nodes and blocks for specific tasks.

The use of the methods described in the article allows the system to control the computational process automatically and solve tasks with a large amount of data, by having a limited amount of local memory of the computational core.

This work is partially funded by RFBR (grants 17-0700478 and 17-07-00324).

\section{REFERENCE}

[1] Dongarra Dzh. Jekzaflopsnoe budushhee superkomp'juterov (Exascale future of supercomputers) // Superkomp'jutery. №1(1). 2010. S. 21-23 (In Russian).

[2] Sterling T. Mnogotochie Sterlinga (Etcetera of Sterling) // Superkomp'juter. №3. 2010. S. 17-20 (In Russian).

[3] Ben Lee, Hurson A.R. Dataflow Architectures and Multithreading. Computer. 1994. Aug. V. 27, no. 8. P. 27-39.

[4] Silc J., Robic B., Ungerer T. Asynchrony in parallel computing: From dataflow to multithreading // Parallel and Distributed Computing Practices. 1998. Vol. 1. № 1. P. 3- 30.

[5] Ivannikov A.D., Levchenko N.N., Okunev A.S., Stempkovsky A.L., Zmejev D.N. Dataflow Computing Model - Perspectives, Advantages and Implementation // Proceedings of IEEE East-West Design \& Test Symposium (EWDTS'2017), Novi Sad, Serbia, Sept 29 - Oct 2, 2017. Pp. 187-190.

[6] Stempkovskij A.L., Levchenko N.N., Okunev A.S., Cvetkov V.V. Parallel'naja potokovaja vychislitel'naja sistema - dal'nejshee razvitie arhitektury i strukturnoj organizacii vychislitel'noj sistemy s avtomaticheskim raspredeleniem resursov (Parallel dataflow computing system the further development of architecture and the structura organization of the computing system with automatic distribution of resources) // Zhurnal «INFORMACIONNYE TEHNOLOGII». 2008. №10. S. 2-7 (In Russian)

[7] Zmejev D.N., Klimov A.V., Levchenko N.N., Okunev A.S., Stempkovsky A.L. The Change of Computation Paradigm and Programming Model - the Future of New Supercomputers // Problems of advanced micro- and nanoelectronic systems development (MES), selected articles of the VII All-Russia Science\&Technology Conference MES-2016, Part II, Moscow, 2017, pp. 40-45. 
[8] Klimov A.V., Levchenko N.N., Okunev A.S., Stempkovsky A.L., Zmejev D.N. The PDCS "Buran" Operating Efficiency Improvement Ways // In bk.: Proceedings of IEEE EAST-WEST DESIGN \& TEST SYMPOSIUM (EWDTS'2016), Yerevan, Armenia, October 14-17, 2016. P. 323-326.

[9] I/O Processor as the Device for Computing Process Management in the PDCS "Buran" // In bk.: Proceedings of IEEE EAST-WEST DESIGN \& TEST SYMPOSIUM (EWDTS'2016), Yerevan, Armenia, October 14-17, 2016. P. 331-334.

[10] Levchenko, N.N., Okunev, A.S.,Yakhontov, D.E. Power Consumption Analysis of Content-Addressable Memories//IEEE EAST-WEST DESIGN \& TEST
SYMPOSIUM 2012, Kharkov, Ukraine, September 14-17, 2012, Symposium Proceedings, p.183-186.

[11] Levchenko, N.N., Okunev, A.S.,Yakhontov, D.E., Zmejev, D.N. Decreasing the Power Consumption of ContentAddressable Memory in the Dataflow Parallel Computing System//IEEE EAST-WEST DESIGN \& TEST SYMPOSIUM 2012, Kharkov, Ukraine, September 14-17, 2012, Symposium Proceedings, p.122-125.

[12] Levchenko N.N., Okunev A.S., Zmejev D.N. Solutions to Problem of CAM Overflow in the Parallel Dataflow Computing System "Buran" // Proceedings of IEEE East-West Design \& Test Symposium (EWDTS'2018), Kazan, Russia, Sept $14-17,2018$, pp. 649-654. 